\title{
High Depressive Symptoms in Previously Undetected Diabetes - 10-Year Follow-Up Results of the Heinz Nixdorf Recall Study
}

\author{
Andrea Icks, (1D) ${ }^{1-3}$ \\ Charlotte Wittgens,' \\ Burkhard Haastert, ${ }^{2,4}$ \\ Karl-Heinz Jöckel, ${ }^{5}$ Miriam Engel, ${ }^{5}$ \\ Raimund Erbel, ${ }^{5}$ Silke Andrich, ${ }^{1,2}$ \\ Johannes Kruse, ${ }^{6}$ Bernd Kulzer, ${ }^{7}$ \\ Norbert Hermanns, ${ }^{7}$ \\ Christian Herder, ${ }^{3,8,9}$ \\ Susanne Moebus, ${ }^{5}$ Andreas Stang, $\mathbb{D}^{5}$ \\ Bernd Kowall ${ }^{5}$ \\ 'Institute for Health Services Research and \\ Health Economics, German Diabetes Center \\ Leibniz Center for Diabetes Research at \\ Heinrich-Heine-University Düsseldorf, \\ Düsseldorf, Germany; ${ }^{2}$ Institute for Health \\ Services Research and Health Economics, \\ Centre for Health and Society, Medical Faculty \\ and University Hospital Düsseldorf, Heinrich- \\ Heine-University Düsseldorf, Düsseldorf, \\ Germany; ${ }^{3}$ German Center for Diabetes \\ Research, Partner Düsseldorf, München- \\ Neuherberg, Germany; ${ }^{4}$ mediStatistica, \\ Neuenrade, Germany; ${ }^{5}$ Institute for Medical \\ Informatics, Biometry and Epidemiology, \\ University Hospital of Essen, Essen, Germany; \\ ${ }_{6}^{6}$ Institute for Psychosomatics and \\ Psychotherapy, University Hospital Gießen, \\ Gießen, Germany; ${ }^{7}$ Research Institute of the \\ Diabetes Academy Mergentheim and Diabetes \\ Centre Mergentheim, Bad Mergentheim, \\ Germany; ${ }^{8}$ Institute for Clinical Diabetology, \\ German Diabetes Center, Leibniz Center for \\ Diabetes Research at Heinrich-Heine- \\ University Düsseldorf, Düsseldorf, Germany; \\ ${ }^{9}$ Division of Endocrinology and Diabetology, \\ Medical Faculty, Heinrich Heine University \\ Düsseldorf, Düsseldorf, Germany
}

Correspondence: Andrea Icks Institute for Health Services Research and Health Economics, German

Diabetes Center, Leibniz Center for

Diabetes Research at Heinrich-Heine-

University Düsseldorf, Auf'm

Hennekamp 65, Düsseldorf, D-40225,

Germany

Tel +492113382400

Fax +49 211 3382677

Email andrea.icks@uni-duesseldorf.de
Aim: To determine the 10-year cumulative incidence of high depressive symptoms in people with diagnosed and, in particular, previously undetected diabetes compared to those without diabetes in a population-based cohort study in Germany.

Materials and Methods: We included 2813 participants (52.9\% men, mean age (SD) 58.9 (7.7) years, $7.1 \%$ diagnosed diabetes, $5.6 \%$ previously undetected diabetes) from the Heinz Nixdorf Recall study. We calculated the odds ratios (OR) with $95 \%$ confidence intervals (CI) using multiple logistic regression analyses for diagnosed and undetected diabetes.

Results: Cumulative 10-year incidences (95\%-CI) of high depressive symptoms in participants with diagnosed diabetes, previously undetected diabetes, and without diabetes were $15.4 \%$ (10.7-21.2), 10.1\% (5.9-15.9), and 12.4\% (11.1-13.8), respectively. Age-sex-adjusted ORs were $1.51(1.01-2.28)$ in participants with diagnosed diabetes compared to those without, 1.40 (0.92-2.12) after adjustment for BMI, physical activity, education, and smoking, and 1.33 (0.87-2.02) after further adjustment for stroke and myocardial infarction. ORs in participants with previously undetected diabetes were 0.96 (0.56-1.65), 0.85 (0.49-1.47), and 0.85 (0.49-1.48), respectively, and lower in men than in women.

Conclusion: As expected, we found an increased odds of developing high depressive symptoms in participants with diagnosed diabetes. However, the odds ratios decreased when we considered comorbidities and other covariates. Interestingly, in participants with previously undetected diabetes, the odds was not increased, even 10 years after detection of diabetes. These results support the hypothesis that high depressive symptoms develop due to diabetes-related burdens and comorbidities and not due to hyperglycemia or hyperinsulinemia.

Keywords: diabetes, undetected diabetes, depressive symptoms, prospective populationbased cohort study

\section{Introduction}

The association between diabetes and prevalent depressive disorders has been frequently analyzed. ${ }^{1}$ Studies show an up to twofold higher prevalence of depressive disorders in persons with diabetes. ${ }^{1}$ Affected people who suffer from a combination of these two health problems have an increased mortality rate, ${ }^{2,3}$ risk of micro- and macrovascular comorbidities ${ }^{4}$ as well as psychological and physical disabilities. ${ }^{5}$ Thus, the combined occurrence of diabetes and depression is of high clinical relevance.

Whether diabetes predicts depressive disorders, vice versa or both, remains disputed. Meta-analyses of prospective longitudinal studies investigating the 
association between diabetes and depression found increased incidences of diabetes in people with depression compared to those without, and increased incidences of depression in people with diabetes compared to those without; however, not all individual studies found these associations. $^{6}$

Moreover, the possible reasons for the association between diabetes and depression are still not sufficiently understood. There are different explanations for the link. One approach assumes that the two diseases are coincidental because they share similar environmental and lifestyle factors, such as socioeconomic deprivation, social adversity, smoking, and reduced physical activity. ${ }^{7}$ Some depressive symptoms are associated with high body-mass index (BMI), poor diet, low levels of physical activity, and smoking, which are also risk factors for the onset of type 2 diabetes. ${ }^{8}$ Another explanation is that depression may develop in association with diabetes-related distress or somatic disorders as (diabetes-related) comorbidities. ${ }^{9}$ Also, depression might be induced by increased activity of the hypothalamic-pituitary-adrenal axis due to biochemical changes associated with diabetes. ${ }^{10}$

If the latter explanation was true, one would expect increased high depressive symptoms not only in diagnosed diabetes but also in previously undetected diabetes. ${ }^{6}$ However, a systematic review and meta-analysis of Nouwen et al including 10 cross-sectional studies has found no difference between the prevalence of high depressive symptoms in individuals with undetected diabetes and without diabetes (OR 0.94; 95\%CI $0.71-1.25) .{ }^{11}$ Prospective studies are rare and show conflicting results: A recent meta-analysis including 5 studies reported that people with newly diagnosed diabetes did not show an increased risk of depressive symptoms in comparison to people with normal glucose metabolism (pooled $\mathrm{RR}=1.07$, 95\%-CI 0.74-1.55). ${ }^{12}$ Another study shows that people with previously diagnosed diabetes had increased odds of depression $(\mathrm{OR}=1.61,95 \%$-CI 1.39-1.87) whereas people with newly diagnosed diabetes had no association with depression. ${ }^{13}$ A more recent large prospective study, however, found no such difference. The authors report no differences in the odds of depression between undiagnosed and diagnosed diabetes $(\mathrm{OR}=1.1,95 \%$-CI $0.7-1.6){ }^{14}$

The analysis of the cross-sectional baseline data of the population-based Heinz Nixdorf Recall study obtained a lower prevalence of high depressive symptoms in men with previously undetected diabetes than in those without diabetes. ${ }^{15}$ In the prospective 5-year follow-up data of this study, the cumulative incidence of high depressive symptoms tended to be lower in participants with undetected diabetes compared to those without diabetes; however, confidence intervals were large (age-sex adjusted OR $=0.72,95 \%$ CI 0.35-1.48). ${ }^{16}$

Considering these findings, we now aimed at estimating the 10-year cumulative incidence of high depressive symptoms in participants with previously undetected as well as diagnosed diabetes and to compare it with that in participants without diabetes. Furthermore, since differences between men and women have often been reported regarding depression, ${ }^{17}$ we stratified our analyses by sex.

\section{Materials and Methods Population}

We analyzed baseline and follow-up data from the populationbased prospective Heinz Nixdorf Recall Study, performed in three adjacent large cities in Western Germany (Essen, Mülheim, Bochum). The design of the study has been described elsewhere in detail. ${ }^{18}$ Briefly, the cohort comprises 4814 randomly selected men and women aged $45-75$ years at baseline (T0). The baseline visits were performed at the Center of Clinical Epidemiology in Essen between 2000 and 2003, the 5-year follow-up (T1) visits between 2005 and 2008, and the 10-year follow-up (T2) visits between 2011 and 2015. The baseline recruitment efficacy proportion was $55.8 \%$, ${ }^{19}$ the 5-year follow-up response proportion $90.2 \%{ }^{20}$ Three thousand eighty-seven participants could be included in the 10year follow-up. The data assessment for all three visits included various medical conditions, conducted by trained study personnel, self-administered questionnaires, and faceto-face interviews for personal risk factor assessment. Comprehensive laboratory tests, anthropometric evaluations, and blood pressure measurements were performed according to standard study protocols. The study was approved by the relevant institutional ethics committees and followed strict internal and external quality assurance protocols. All participants gave their written informed consents.

Exclusion criteria for the analysis were participants with either missing or positive values for our primary depression variable at T0. From the 4814 participants at baseline, for the present analysis, 848 participants were excluded because of missing data of depression history, and additional 147 participants because of the unknown status of depressive symptoms at baseline (missing in the assessment using the CES-D as a specific instrument, for description see below). The reason for the large number of participants without information on their depression history is that for some months, 
because of limited capacities in the study center, only an abbreviated interview without reference to depression history was performed. Participants without data on depression history were well comparable to the whole study population (Table S1, Appendix). Four hundred and nine participants were excluded due to a self-reported diagnosis of depression in the medical history at baseline, as were another 244 participants who were identified with depressive symptoms in the baseline data assessment using the CES-D scale. Three thousand one hundred and sixty-six participants remained. Another 350 participants without depression data in the follow-up and finally three persons without relevant data in the follow-up were excluded, yielding the final study sample of $\mathrm{N}=2813$ participants for the main analysis. Figure 1 gives an overview of the participant selection of the study populations.

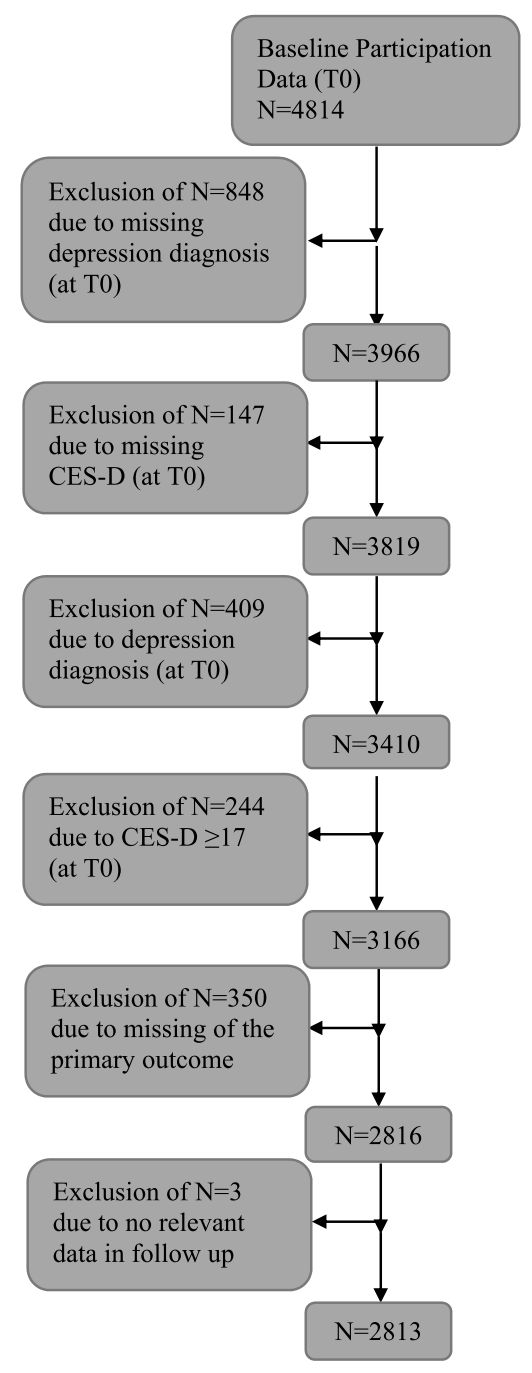

Figure I Flow chart: overview of participant selection criteria for study populations.

\section{Variables}

\section{Outcome: High Depressive Symptoms}

We defined high depressive symptoms by two criteria: (1) a positive result in the 15 -item short form of the Center for Epidemiologic Studies Depression scale (CES-D), a commonly used screening instrument, ${ }^{21}$ and (2) a selfreported depression diagnosis. Ad (1): The CES-D follows the ICD-10 diagnoses. ${ }^{22}$ Participants received the CES-D at baseline, as well as at 5-year and 10-year follow-up. Ad (2): At baseline, participants were asked if they had a depression diagnosis in their medical history. At 5-year follow-up (T1) and at 10-year follow-up (T2), they were asked whether they have had a diagnosis of depression at any point in time during the preceding 5 years. Participants were considered as having developed high depressive symptoms when they gave a selfreport of diagnosed depression or when they had a CES-D score $\geq 17$ during at least one follow-up visit. For 40 participants at T1, only CES-D values from telephone interviews could be used; information on depression diagnoses did not form part of the telephone interview.

\section{Exposure Assessment: Previously Undetected and Diagnosed Diabetes}

We considered participants to have diagnosed diabetes mellitus if clinician-documented diagnosis of diabetes was reported in the medical history interview or if glucose-lowering drugs were taken. ${ }^{16}$ Previously undetected diabetes at baseline was defined by the combination of the following factors: (i) fasting glucose $\geq 7.0 \mathrm{mmol} / 1(61 \%$, $77 \%$, and $80 \%$ of study participants were in a fasting state at baseline and the two follow-ups, respectively, with fasting state defined as fasting for 8 hours) or random blood glucose $\geq 11.1 \mathrm{mmol} / \mathrm{l}^{23}$ (the remaining participants were in a non-fasting state), and (ii) participants had not reported a diabetes diagnosis and were not taking any glucose-lowering agents.

\section{Covariates}

Covariates known to modify depressive symptoms that were also associated with diabetes or potential mediators were selected almost identically to that of the 5-year follow-up analyses. ${ }^{16}$ For the previous as well as for the current analysis, we identified possible covariates from clinical experience and the literature and discussed them carefully prior to the analyses. We therefore included age (in years) and sex as covariates. Furthermore, we included BMI $\left(>30 \mathrm{~kg} / \mathrm{m}^{2}\right.$ versus $\left.\leq 30 \mathrm{~kg} / \mathrm{m}^{2}\right)$, physical activity (sports: no vs yes), myocardial infarction, stroke 
occurrence, and education status according to the International Standard Classification of Education' (ordinal with values $1-4),{ }^{24}$ smoking status (3 categories: former or current smoker vs nonsmoker), and, in additional models, C-reactive protein (CRP) and adiponectin (log values because of skewed distribution) as biomarkers of inflammation. All these variables were documented at baseline. Persons with diabetes might have a lower chance of participation at 10-year follow-up (eg because of death), and thus a lower chance of reporting depression. To close this pathway, we also considered participation at 10-year follow-up as additional covariate in sensitivity analyses.

\section{Statistical Analyses}

Descriptive statistics were used to summarize the baseline characteristics, stratified by diabetes status. The cumulative incidences of high depressive symptoms after ten years were estimated as proportions of participants developing high depressive symptoms during follow-up with 95\% confidence intervals (CI) (Pearson-Clopper) for participants with diagnosed diabetes, with previously undetected diabetes, and without diabetes at baseline.

Multiple logistic regression models were fitted to estimate odds ratios (OR) and 95\% confidence intervals for the association of high depressive symptoms during follow-up (cumulative from T0 to T2) (dependent variable) and diabetes status at baseline (previously undetected/ diagnosed/no diabetes as independent variable) separately on both corresponding subpopulations of participants with diagnosed resp. undetected diabetes. Further covariates were included as independent variables in the models. Four separate models were calculated on both subpopulations. Model 1 was only adjusted for age and sex. Model 2 included further covariates (education, physical activity, BMI, and smoking). Model 3 additionally included the variables stroke and myocardial infarction to discuss their role as potential mediators. Model 4 was extended by including biomarkers of inflammation which may also be mediators (stroke and myocardial infarction excluded). Furthermore, the main models were fitted separately for women and men.

For statistical analyses, SAS version 9.4 was used.

\section{Sensitivity Analyses}

A sensitivity analysis was computed excluding participants with undetected diabetes at baseline if a diagnosis was not confirmed at $\mathrm{T} 1$ or $\mathrm{T} 2$.
In another sensitivity analysis (data not shown in detail), participation at the second follow-up was considered as an additional independent variable in models 2, 3 and 4 to investigate a possible bias from the lack of information on depression at T2 (censoring, eg, due to death) between the participants with detected/undetected diabetes and the reference group without diabetes.

\section{Ethic Statement}

Approval was obtained from the Ethics Committee of the University Clinic Essen.

We confirm that the data accessed complies with relevant data protection and privacy regulations.

\section{Results}

\section{Characteristics of the Study Cohort}

The characteristics of our study cohort $(n=2813)$ stratified by diabetes status are presented in Table 1. About half of the study population was male, and the mean age was about 60 years. Participants with diabetes were older, more frequently male, had a higher BMI, and were less physically active as participants without diabetes. They also more often reported stroke and myocardial infarction at baseline. Participants with previously undetected diabetes showed similar characteristics to those with diagnosed diabetes.

Tables S2 and S3 in the appendix present sex-stratified characteristics. As expected for this age cohort in Germany, women were less educated, less frequently smokers, more physically active, and had fewer cardiovascular diseases compared to men.

\section{Cumulative Incidences of Depressive Symptoms}

Mean follow-up duration was $9.2 \pm 2.2$ years. A total of 351 participants developed high depressive symptoms during follow-up $(31,16$, and 304 participants had previously diagnosed diabetes, undetected diabetes, and no diabetes, respectively). The 10-year cumulative depression incidences of the primary outcome were $15.4 \%$ (95\%-CI 10.7-21.2\%) in those with diagnosed diabetes, $10.1 \%(95 \%$-CI $5.9-15.9 \%)$ in those with undetected diabetes, and $12.4 \%$ (95\%-CI 11.1-13.8\%) in participants with no diabetes (Table 2). 
Table I Baseline Participant Characteristics, Stratified by Diabetes Status

\begin{tabular}{|c|c|c|c|}
\hline & Diagnosed Diabetes & Undetected Diabetes & No Diabetes \\
\hline & $(n=20 I)$ & $(n=158)$ & $(n=2454)$ \\
\hline Age, mean (SD), y & $61.1(7.4)$ & $60.3(7.5)$ & $58.6(7.6)$ \\
\hline Male (\%) & 65.7 & 72.8 & 50.6 \\
\hline \multicolumn{4}{|l|}{ Education: years of school \% $(95 \%-\mathrm{Cl})^{\mathrm{a}}$} \\
\hline$\leq 10$ & II.4 (7.4-16.7) & $8.2(4.5-13.7)$ & $8.5(7.5-9.7)$ \\
\hline $11-13$ & $54.7(47.6-6 \mid .7)$ & $55.7(47.6-63.6)$ & $55.2(53.2-57.2)$ \\
\hline $14-17$ & $23.9(18.2-30.4)$ & $28.5(21.6-36.2)$ & $23.8(22.1-25.5)$ \\
\hline$\geq 18$ years & $10.0(6.2-14.9)$ & $7.6(4.0-12.9)$ & $12.5(\mid 1.2-13.9)$ \\
\hline No regular physical exercise \% $(95 \%-\mathrm{Cl})$ & $54.7(47.6-61.7)$ & $58.2(50.1-66.0)$ & $44.4(42.4-46.4)$ \\
\hline BMI, mean (SD), $\left(\mathrm{kg} / \mathrm{m}^{2}\right)^{\mathrm{b}}$ & $30.1(5.1)$ & $30.6(5.2)$ & $27.3(4.2)$ \\
\hline $\mathrm{BMI}>30 \mathrm{~kg} / \mathrm{m}^{2}(95 \%-\mathrm{Cl})(\%)^{\mathrm{b}}$ & $43.0(36.0-50.2)$ & $51.0(42.9-59.0)$ & $21.0(19.4-22.7)$ \\
\hline HbAlc (mean, SD) $(\%)^{c}$ & $7.0(1.3)$ & $6.3(1.6)$ & $5.3(0.5)$ \\
\hline CRP, median (quartiles) ${ }^{d}$ & $0.20(0.08-0.43)$ & $0.17(0.09-0.36)$ & $0.13(0.07-0.28)$ \\
\hline Adiponectin, median (quartiles) ${ }^{\mathrm{e}}$ & $6135(4 \mid 47-10,148)$ & $5390(347 I-8394)$ & $820 I(5532-12,373)$ \\
\hline \multicolumn{4}{|l|}{ Smoking: \% $(95 \%-\mathrm{Cl})^{f}$} \\
\hline Former smoker & $41.8(34.9-48.9)$ & $43.7(35.8-51.8)$ & $35.5(33.6-37.4)$ \\
\hline Current smoker & $21.9(16.4-28.3)$ & $25.9(19.3-33.5)$ & $21.8(20.2-23.5)$ \\
\hline \multicolumn{4}{|l|}{ Comorbidities \% (95\%-Cl) } \\
\hline CVD & $14.4(9.9-20.1)$ & $10.1(5.9-15.9)$ & $4.5(3.7-5.4)$ \\
\hline Myocardial infarction ${ }^{g}$ & $9.0(5.4-13.9)$ & $7.7(4.0-13.1)$ & $3.1(2.4-3.8)$ \\
\hline Stroke $^{\mathrm{h}}$ & $8.0(4.6-12.7)$ & $1.3(0.2-4.5)$ & $1.5(1.0-2.0)$ \\
\hline Participation at 2 nd Follow-Up after 10 yrs \% $(95 \%-C l)$ & $64.7(57.6-71.3)$ & $63.9(55.9-71.4)$ & $77.5(75.8-79.2)$ \\
\hline Diabetes duration, mean (SD), $y^{i}$ & $9.8(11.5)$ & & \\
\hline \multicolumn{4}{|l|}{ Diabetes-related comorbidities \% $(95 \%-\mathrm{Cl})$} \\
\hline Retinopathy ${ }^{j}$ & $10.6(6.6-15.9)$ & & \\
\hline Blindness $^{k}$ & $0.5(0.0-2.9)$ & & \\
\hline Proteinuria $^{\prime}$ & $17.4(11.7-24.5)$ & & \\
\hline Renal failure $^{m}$ & $2.1(0.6-5.2)$ & & \\
\hline Renal replacement therapy ${ }^{n}$ & $1.0(0.1-3.7)$ & & \\
\hline Neuropathy ${ }^{\circ}$ & $31.6(25.0-38.7)$ & & \\
\hline Amputation $^{\mathrm{P}}$ & $2.1(0.6-5.2)$ & & \\
\hline
\end{tabular}

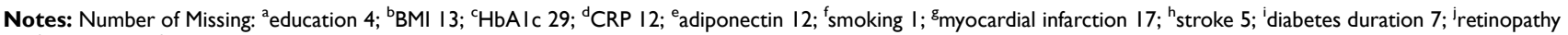
12; " blindness 8; 'proteinuria 52; ${ }^{\mathrm{m}}$ renal failure 8 ; ${ }^{\mathrm{n}}$ renal replacement therapy 8 ; ${ }^{\circ}$ neuropathy $\mathrm{II}$; ${ }^{\text {Pamputation } 8 .}$

\section{Odds Ratios from Multiple Regression Analysis}

In the sex-age-adjusted logistic regression analysis (Table 2 and $\underline{\mathrm{S} 4}$ in the appendix including detailed results for all covariates) diagnosed diabetes was associated with depressive symptoms (1.51 (95\%-CI 1.01-2.28)). After adjustment for the other confounders (model 2), the association between diagnosed diabetes and depressive symptoms was somewhat weaker compared to the sexage adjusted model (OR 1.40 (95\%-CI $0.92-2.12)$ ). Including the potential mediators stroke and myocardial infarction as covariates in model 3 further reduced the OR of diagnosed diabetes $(1.33(0.87-2.02))$. When markers of inflammation were included instead of stroke and myocardial infarction (model 4), ORs were similar to model 2. 
Table 2 Multiple Logistic Regression Models Analyzing the Relationship Between Diagnosed and Undetected Diabetes and the Development of High Depressive Symptoms: The Heinz Nixdorf Recall Study

\begin{tabular}{|c|c|c|c|c|c|c|}
\hline & \multirow[t]{2}{*}{$\mathbf{N}$} & Incident Cases n (\%) & \multirow{2}{*}{$\begin{array}{c}\text { Model I: OR } \\
(95 \%-\mathrm{Cl})\end{array}$} & \multirow{2}{*}{$\begin{array}{c}\text { Model 2: OR } \\
(95 \%-\mathrm{Cl})\end{array}$} & \multirow{2}{*}{$\begin{array}{l}\text { Model 3: OR } \\
(95 \%-\mathrm{Cl})\end{array}$} & \multirow{2}{*}{$\begin{array}{c}\text { Model 4: OR } \\
(95 \%-\mathrm{Cl})\end{array}$} \\
\hline & & (\% 95\%-Cl) & & & & \\
\hline Diagnosed diabetes & 201 & $\begin{array}{r}3 \mid(15.4 \%) \\
(|0.7-2| .2 \%)\end{array}$ & $1.51(1.01-2.28)$ & $1.40(0.92-2.12)$ & $1.33(0.87-2.02)$ & $1.46(0.96-2.21)$ \\
\hline Undetected diabetes & 158 & $\begin{array}{r}16(10.1 \%) \\
(5.9-15.9 \%)\end{array}$ & $0.96(0.56-1.65)$ & $0.85(0.49-1.47)$ & $0.85(0.49-1.48)$ & $0.76(0.43-1.36)$ \\
\hline No diabetes (ref) & 2454 & $\begin{array}{r}304(12.4 \%) \\
(11.1-13.8 \%)\end{array}$ & 1.00 & 1.00 & 1.00 & 1.00 \\
\hline \multicolumn{7}{|l|}{ Men } \\
\hline Diagnosed diabetes & 132 & $\begin{array}{r}18(13.6 \%) \\
(8.3-20.7 \%)\end{array}$ & I.67 (0.98-2.86) & $1.59(0.92-2.76)$ & $\mathrm{I} .49(0.85-2.6 \mathrm{I})$ & $1.69(0.97-2.93)$ \\
\hline Undetected diabetes & 115 & $\begin{array}{r}8(7.0 \%) \\
(3.1-13.2 \%)\end{array}$ & $0.77(0.37-1.63)$ & $0.70(0.33-1.50)$ & $0.69(0.32-1.49)$ & $0.76(0.35-1.63)$ \\
\hline No diabetes (ref) & 1242 & $\begin{array}{r}115(9.3 \%) \\
(7.7-11.0 \%)\end{array}$ & 1.00 & 1.00 & 1.00 & 1.00 \\
\hline \multicolumn{7}{|l|}{ Women } \\
\hline Diagnosed diabetes & 69 & $\begin{array}{r}13(18.8 \%) \\
(10.4-30.1 \%)\end{array}$ & $1.34(0.7 \mid-2.5 I)$ & $1.24(0.65-2.35)$ & $1.19(0.63-2.28)$ & $1.26(0.66-2.40)$ \\
\hline Undetected diabetes & 43 & $\begin{array}{r}8(18.6 \%) \\
(8.4-33.4 \%)\end{array}$ & $1.29(0.59-2.83)$ & $\mathrm{I} .14(0.5 \mathrm{I}-2.53)$ & I.I8 (0.53-2.62) & $0.83(0.34-2.04)$ \\
\hline No diabetes (ref) & 1212 & $\begin{array}{r}189(15.6 \%) \\
(13.6-17.8 \%)\end{array}$ & 1.00 & 1.00 & 1.00 & 1.00 \\
\hline
\end{tabular}

Notes: Different models for diagnosed and undetected diabetes on corresponding subpopulations including reference population. Model I: adjusted for age and sex. Model 2: Model I + additional adjustment for BMI, physical activity, smoking, education (only confounders). Model 3: Model $2+$ additional adjustment for myocardial infarction and stroke (confounders + Ml + stroke). Model 4: Model $2+$ additional adjustment for log-CRP and log-adiponectin (confounders + immunological parameters). Missing: maximal 16 (model 2.3) or 56 (model 4) participants excluded because of missing in each model for diagnosed/undetected diabetes.

Abbreviations: $\mathrm{OR}$, odds ratio; $\mathrm{Cl}$, confidence interval.

Undetected diabetes was not or barely associated with depressive symptoms - sex- or age-adjusted OR versus no diabetes: 0.96 (95\%-CI 0.56-1.65) after further adjustment in model 2 OR 0.85 (0.49-1.47).

\section{Sex-Specific Results}

Regarding sex-stratified analyses (Table 2), cumulative incidences of high depression symptoms were 13.6\% (95\%-CI 8.3-20.7\%) for males with known diabetes, $7.0 \%(3.1-13.2 \%)$ for males with undetected diabetes, $9.3(7.7-11.0 \%)$ for males without diabetes, and $18.8 \%$ (10.4-30.1\%) for females with known diabetes, $18.6 \%$ $(8.4-33.4 \%)$ for females with undetected diabetes, $15.6 \%$ (13.6-17.8\%) for females without diabetes.

For males, the age-adjusted odds ratio in model 1 for known diabetes was $1.67(0.98-2.86)$, the multiple adjusted OR was $1.59(0.92-2.76)$ in model 2, and 1.49 (0.85-2.61) in model 3 . The corresponding ORs for undetected diabetes were age-adjusted 0.77 (0.37-1.63), 0.70 (0.33-1.50) in model 2, and $0.69(0.32-1.49)$ in model 3.

For females, ORs for known diabetes were ageadjusted 1.34 (0.71-2.51), $1.24(0.65-2.35)$ in model 2, and $1.19(0.63-2.28)$ in model 3. Female ORs for undetected diabetes were $1.29(0.59-2.83)$ in model $1,1.14$ (0.51-2.53) in model 2, and $1.18(0.53-2.69)$ in model 3.

\section{Sensitivity Analyses}

After excluding 40 out of 158 participants with undetected diabetes without any confirmation of the diabetes diagnosis in the follow-up, 118 participants with confirmed diagnosis of initially undetected diabetes remained in the study population. The ORs of undetected diabetes versus no 
diabetes were $1.16(95 \%$-CI $0.65-2.07)$ in model 1 , and 1.01 (0.56-1.82) in model 2 (Table S5, Appendix).

In the sensitivity analyses including participation at the second follow-up as an additional independent variable (in models 2, 3 and 4), a small increase of the ORs was observed in all models, for diagnosed diabetes from 1.40, 1.33, and 1.46 (models 2, 3, 4 without T2 participation) to 1.44, 1.36, 1.51 (models 2, 3, 4 including T2 participation), for undetected diabetes from $0.85,0.85,0.76$ to 0.88 , $0.89,0.79$. This small increase might correspond to the above-mentioned possible bias from lower T2 participation in the diabetes subgroups, but it would not change the interpretation.

\section{Discussion}

\section{Main Study Findings and Comparison to Other Studies}

Our study is, to the best of our knowledge, the first to analyze the incidence of high depressive symptoms in individuals with diagnosed and previously undiagnosed diabetes mellitus versus participants without diabetes in a prospective study design over a 10 -year period. We found an increased odds for developing depression in people with diagnosed diabetes at baseline, as expected. In contrast, in people with undetected diabetes at baseline, the risk of developing depression was not increased, even after 10 years.

A number of studies analyzed the incidence of depression in diagnosed diabetes and found it to be $15-24 \%$ increased. ${ }^{6-10}$ Our study is well in line with these findings. However, up to now, only five studies have analyzed the incidence of depression in people with previously undetected diabetes, with conflicting results. ${ }^{11}$ Whereas in the study by Golden the incidence tended to be lower compared to people without diabetes, the studies by Pieper, Demakakos and Okumiya found an increased risk of depression in people with previously undetected diabetes. ${ }^{25-28}$ In our 5-year follow-up study, ${ }^{16}$ the incidence tended to be lower compared to people without diabetes, but after ten years, the incidences were comparable. However, one must consider that compared to our previous analysis on 5-year follow-up, we changed the definition of our primary outcome as we assessed high depressive symptoms using a combined variable of a CES-D $\geq 17$ or diagnosis of depression in the medical history without a medical validation. This definition means a higher sensitivity to symptoms of depression during followup, but the specificity is slightly reduced by the change.
Considering differences between the sexes, which have been analyzed in this study for the first time, one has to keep in mind the low case numbers, particularly among women. Nevertheless, there may be differences: Whereas women with previously undetected diabetes seem to have an increased risk of developing depression compared to those without diabetes at baseline, in men with previously undetected diabetes, this risk appears to be lower. A similar finding has been observed in our previous studies: in the cross-sectional analysis, men with undetected diabetes had a lower prevalence of depression compared to men without diabetes (OR 0.30; 95\%-CI 0.13-0.70). ${ }^{15}$ In the 5-year follow-up, the risk of developing depression in conjunction with previously undetected diabetes was 0.42 (95\%-CI 0.13-1.37) in men, and $0.96(0.37-2.48)$ in women (re-analysis from the 5-year follow-up). ${ }^{16}$ A possible reason could be that men feel less concerned about a diabetes diagnosis than women. In addition, past epidemiological studies have shown consistently higher depression prevalence and rates in females than in males. $^{17,29}$ The reasons for this are not fully understood. Besides hormonal differences, a reporting bias is also often discussed: men might be less likely to speak about emotional experiences than women.

Another interesting variable is the age. In the study by Demakakos et al, it appears that younger subjects $(<65$ years) with diagnosed diabetes tend to have a higher risk of incident depressive symptoms compared to older subjects. ${ }^{25}$ We performed an explorative analysis stratifying for age. In our analyses, younger people ( $<65$ years) with diagnosed diabetes had a higher risk of developing depression compared to younger people without diabetes (adjusted OR 1.64; 95\%-CI 0.99-2.71), whereas in the older age group, there seem to be no differences between those with and without diabetes (OR 1.13;0.52-2.43). In contrast, in people with previously undetected diabetes, the younger group ( $<65$ years) tended to have a lower risk compared to those without diabetes (OR $0.75 ; 0.38-1.48$ ), whereas in the older age group, there seemed to be no difference (OR $\geq 65$ years: $1.05 ; 0.41-2.65)$.

\section{Discussion and Implications}

In our study, participants with previously undetected diabetes have been informed about their elevated blood glucose measures and recommended to consult their doctors. Hence, their diabetes was not longer undetected during the follow-up period. The question arises why these participants still seemed to have a lower risk for 
developing high depressive symptoms than those with previously diagnosed diabetes. This may be explained by the hypothesis that depressive symptoms are associated with the burden of treatment and diabetes-related complications, as discussed by Tong in his meta-analysis. The case of our newly diagnosed participants speaks in favor of the hypothesis that depressive symptoms only increase among those with clinically identified diabetes, but not among those with previously undetected diabetes or pre-diabetes. ${ }^{11}$ The results of our 5-year follow-up point into the same direction. ${ }^{16}$ The severity and symptoms of diabetes may also play a role. People with undiagnosed diabetes might have fewer diabetes-related complaints, show fewer diabetic complications, and have a lower HbA1c compared to people with diagnosed diabetes even in a time period over 10 years, which might explain why their diabetes-related burden is lighter, which plays a role in the development of depressive symptoms.

\section{Study Limitations and Strengths}

The study has several limitations: First, a high number of participants died during follow-up, leading to a risk of bias. However, we performed a sensitivity analysis including participation in $\mathrm{T} 2$ as an additional independent variable in the models with only minor changes of the results. Second, misclassification may have occurred, in particular in participants with previously undetected diabetes. However, when we estimated 10-year survival probability in the three groups using Kaplan-Meier curves, we found it to be lowest in participants with diagnosed diabetes, followed by those with previously undetected diabetes, both markedly lower than in those without diabetes (79.3\% (95\%-CI 73.6-83.9), $83.2 \%$ (77.0-87.9), and 91.4\% (90.3-92.4), respectively). This observation supports the correct classification of the diabetes status. Third, the sample size, especially that of patients with undiagnosed diabetes, was small, resulting in imprecise estimates with large confidence intervals. The results of multiple subgroup analysis were also limited by the small sample size. Fourth, we were not able to consider diabetes duration and diabetic-specific complications in people with undetected diabetes, since this information was not available. However, we considered BMI, HbAlc as well as the presence of CVD, myocardial infarction, and stroke. Furthermore, stroke and myocardial infarction were discussed as potential mediators. However, a formal mediator analysis was not performed because this was only a secondary question; complete control of confounders between exposition, mediator and outcome cannot be assured.

The strengths of the study are as follows: We assessed high depressive symptoms using a common and well-established instrument. The CES-D has been used frequently to analyze the association of diabetes and depressive symptoms or depression. ${ }^{21}$ The HNR study includes carefully assessed variables and is well-performed and well-investigated. The number of drop-outs was low, and the follow-up participation over two observation points was high.

\section{Conclusion}

To the best of our knowledge, this is the first prospective study including a 10-year follow-up that investigates the risk of developing high depressive symptoms in participants with previously undetected diabetes as well as diagnosed diabetes compared to participants without diabetes. Furthermore, it is the first study stratifying by sex. As expected, we found an increased odds of developing high depressive symptoms in participants with diagnosed diabetes. However, the odds ratios decreased when we considered comorbidities and other covariates. In participants with previously undetected diabetes, the odds were not increased, even 10 years after detection of diabetes, and there may be differences between men and women. These results support the hypothesis that high depressive symptoms develop due to diabetes-related burden and comorbidities rather than due to hyperglycemia or hyperinsulinemia directly.

\section{Acknowledgments}

We thank the investigative group, in particular U. Roggenbuck, U. Slomiany, E.M. Beck, A. Öffner, S. Münkel, M. Bauer, S. Schrader, R. Peter, and H. Hirche, and the study staff of the Heinz Nixdorf Recall Study as well as all study participants. Furthermore, we would like to thank Jeremy Groves and Alexandra Berlina for proofreading the manuscript.

\section{Author Contributions}

Project leader, development of the conception and design of the study: Andrea Icks. Project management: Andrea Icks, Charlotte Wittgens, Biometric/statistical expertise: Burkhard Haastert, Andreas Stang. Contributions to the study design and methodology: Burkhard Haastert, Andreas Stang, Karl-Heinz Jöckel. Guarantors of the Heinz Nixdorf Research Study: Raimund Erbel, KarlHeinz Jöckel, Susanne Moebus. Additional clinical expertise: Johannes Kruse, Norbert Hermanns, Bernd 
Kulzer. Reviewing of the manuscript; substantial contributions to subsequent drafts: Andrea Icks, Charlotte Wittgens, Burkhard Haastert, Karl-Heinz Jöckel, Miriam Engel, Raimund Erbel, Silke Andrich, Johannes Kruse, Bernd Kulzer, Norbert Hermanns, Christian Herder, Susanne Moebus, Andreas Stang, Bernd Kowall. Approval of the final manuscript: Andrea Icks, Charlotte Wittgens, Burkhard Haastert, Karl-Heinz Jöckel, Miriam Engel, Raimund Erbel, Silke Andrich, Johannes Kruse, Bernd Kulzer, Norbert Hermanns, Christian Herder, Susanne Moebus, Andreas Stang, Bernd Kowall. Data analysis: Burkhard Haastert. Contribution of reagents/ materials/analysis tools: Christian Herder. Writing: Andrea Icks.

All authors made substantial contributions to the conception and design, the acquisition, analysis or interpretation of data; they took part in drafting the article or revising it critically for important intellectual content; agreed to submit to the current journal; gave final approval of the version to be published and agree to be accountable for all aspects of the work.

\section{Funding}

The study was supported by institutional funding (German Center for Diabetes Research) of the German Ministry of Health and by the Ministry of Culture and Science of North-Rhine Westphalia. It was further funded by the German Ministry of Education and Research as part of the German Center for Diabetes Research. The Heinz Nixdorf Study was funded by the Heinz Nixdorf Foundation (Chairman: Dr jur. G. Schmidt). This study is also supported by the German Ministry of Education and Science (BMBF), which transferred the monitoring of the study to the German Aerospace Center [Deutsches Zentrum für Luft- und Raumfahrt (DLR)], Bonn, Germany.

\section{Disclosure}

Prof. Dr. Christian Herder reports grants from SanofiAventis outside the submitted work. The authors declare that they have no other potential conflicts of interest for this work.

\section{References}

1. Ali S, Stone MA, Peters JL, Davies MJ, Khunti K. The prevalence of co-morbid depression in adults with type 2 diabetes: a systematic review and meta-analysis. Diabet Med. 2006;23(11):1165-1173. doi:10.1111/j.1464-5491.2006.01943.x
2. Katon W, Fan MY, Unutzer J, Taylor J, Pincus H, Schoenbaum M. Depression and diabetes: a potentially lethal combination. J Gen Intern Med. 2008;23(10):1571-1575. doi:10.1007/s11606-008-0731-9

3. Zhang X, Norris SL, Gregg EW, Cheng YJ, Beckles G, Kahn HS. Depressive symptoms and mortality among persons with and without Diabetes. Am J Epidemiol. 2005;161(7):652-660. doi:10.1093/aje/ kwi089

4. Bruce DG, Davis WA, Starkstein SE, Davis TME. A prospective study of depression and mortality in patients with type 2 diabetes: the Fremantle Diabetes Study. Diabetologia. 2005;48 (12):2532-2539. doi:10.1007/s00125-005-0024-3

5. Black SA, Markides KS, Ray LA. Depression predicts increased incidence of adverse health outcomes in older Mexican Americans with type 2 diabetes. Diabetes Care. 2003;26(10):2822-2828. doi: $10.2337 /$ diacare.26.10.2822

6. Nouwen A, Winkley K, Twisk J, et al.; European Depression in Diabetes (EDID) Research Consortium. Type 2 diabetes mellitus as a risk factor for the onset of depression: a systematic review and meta-analysis. Diabetologia. 2010;53(12):2480-2486. doi:10.1007/ s00125-010-1874-x

7. Moulton CD, Pickup JC, Ismail K. The link between depression and diabetes: the search for shared mechanisms. Lancet Diabetes Endocrinol. 2015;3(6):461-471. doi:10.1016/S2213-8587(15)00134-5

8. Katon WJ, Russo JE, Heckbert SR, et al. The relationship between changes in depression symptoms and changes in health risk behaviors in patients with diabetes. Int $J$ Geriatr Psychiatry. 2010;25 (5):466-475. doi:10.1002/gps.2363

9. Gonzales JS, Fisher L, Polonsky WH. Depression in diabetes: have we been missing something important? Diabetes Care. 2011;34:236-239. doi:10.2337/dc10-1970

10. Mezuk B, Eaton WW, Albrecht S, Golden SH. Depression and type 2 diabetes over the lifespan: a meta-analysis. Diabetes Care. 2008;31 (12):2383-2390. doi:10.2337/dc08-0985

11. European Depression in Diabetes (EDID) Research Consortium; Nouwen A, Nefs G, Caramlau I, et al. Prevalence of depression in individuals with impaired glucose metabolism or undiagnosed diabetes: a systematic review and meta-analysis of the European Depression in Diabetes (EDID) Research Consortium. Diabetes Care. 2011;34(3):752-762. doi:10.2337/dc10-1414.

12. Tong A, Wang X, Li F, Xu F, Li Q, Zhang F. Risk of depressive symptoms associated with impaired glucose metabolism, newly diagnosed diabetes, and previously diagnosed diabetes: a meta-analysis of prospective cohort studies. Acta Diabetol. 2016;53(4):589-598. doi:10.1007/s00592-016-0845-1

13. Sun JC, Xu M, Lu JL, et al. Associations of depression with impaired glucose regulation, newly diagnosed diabetes and previously diagnosed diabetes in Chinese adults. Diabet Med. 2015;32(7):935-943. doi:10.1111/dme.12649

14. Meurs M, Roest AM, Wolffenbuttel BHR, Stolk RP, de Jonge P, Rosmalen JGM. Association of depressive and anxiety disorders with diagnosed versus undiagnosed diabetes: an epidemiological study of 90,686 participants. Psychosom Med. 2016;78(2):233-241. doi:10.1097/PSY.0000000000000255

15. Icks A, Kruse J, Dragano N, et al. Are symptoms of depression commoner in diabetes? Results from the Heinz Nixdorf Recall study. Diabet Med. 2008;25(11):1330-1336. doi:10.1111/j.14645491.2008.02585.x

16. Icks A, Albers B, Haastert B, et al. Risk for high depressive symptoms in diagnosed and previously undetected diabetes: 5-year follow-up results of the Heinz Nixdorf Recall Study. PLoS One. 2013;8(2):e56300. doi:10.1371/journal.pone.0056300

17. Angst J, Gamma A, Gastpar M, Lépine JP, Mendlewicz J, Tylee A. Gender differences in depression. Epidemiological findings from the European DEPRES I and II studies. Eur Arch Psychiatry Clin Neurosci. 2002;252:201-209. doi:10.1007/s00406-002-0381-6 
18. Schmermund A, Möhlenkamp S, Stang A, et al. Assessment of clinically silent atherosclerotic disease and established and novel risk factors for predicting myocardial infarction and cardiac death in healthy middle-aged subjects: rationale and design of the Heinz Nixdorf RECALL Study. Am Heart J. 2002;144:212-218. doi:10.1067/mhj.2002.123579

19. Stang A, Moebus S, Dragano N, et al. Baseline recruitment and analyses of nonresponse of the Heinz Nixdorf Recall Study: identifiability of phone numbers as the major determinant of response. Eur J Epidemiol. 2005;20:489-496. doi:10.1007/s10654-005-5529-z

20. Erbel R, Möhlenkamp S, Moebus S, et al. Coronary risk stratification, discrimination, and reclassification improvement based on quantification of subclinical coronary atherosclerosis. The Heinz Nixdorf Recall Study. J Am Coll Cardiol. 2010;56:1397-1406. doi:10.1016/ j.jacc. 2010.06 .030

21. Bekman ATF, Deeg DJH, Van Limbeek J, et al. Criterion validity of the Center for Epidemiologic Studies Depression scale (CES-D): results from a community-based sample of older subjects in the Netherlands. Psychol Med. 1997;27:231-235. doi:10.1017/S0033291796003510

22. Lehr D, Hillert A, Schmitz E, Sosnowsky N. Screening depressiver Störungen mittels Allgemeiner Depressions-Skala (ADS-K) und State-Trait Depressions Scales (STDS-T). Eine vergleichende Evaluation von Cut-Off-Werten. Diagnostica. 2008;54:61. doi:10.1026/0012-1924.54.2.61

23. World Health Organization. Definition, Diagnosis and Classification of Diabetes Mellitus and Its Complications. Report of the WHO Consultation. Part 1: Diagnosis and Classification of Diabetes Mellitus. Geneva: World Health Organization, Department of Noncommunicable Disease Surveillance; 1999.
24. UNESCO. International standard classification of education. In: Hoffmeyer-Zlotnik JHP, Wolf C, editors. Advances in CrossNational Comparisons. New York: Kluwer; 2003:195-220.

25. Golden SH, Lazo M, Carnethon M, et al. Examining a bidirectional association between depressive symptoms and diabetes. JAMA. 2008;299(23):2751-2759. doi:10.1001/jama.299.23.2751

26. Pieper L, Dirmaier J, Klotsche J, et al. Longitudinal associations between depressive symptoms and type 2 diabetes and their impact on mortality in primary care patients. Bundesgesundheitsblatt Gesundheitsforschung Gesundheitsschutz. 2011;54(1):98-107. doi:10.1007/s00103-010-1181-1

27. Demakakos P, Zaninotto P, Nouwen A. Is the association between depressive symptoms and glucose metabolism bidirectional? Evidence from the English Longitudinal Study of Ageing (ELSA). Psychosom Med. 2014;76(7):555. doi:10.1097/PSY.0000000 000000082

28. Okumiya K, Fujisawa M, Sakamoto R, et al. Effect of early diagnosis and lifestyle modification on depressive symptoms in communitydwelling elderly adults with glucose intolerance: 5-year longitudinal study. J Am Geriatr Soc. 2015;63(2):393-395. doi:10.1111/jgs.13269

29. de la Torre JA, Vilagutcd G, Martínac V, Molinaa AJ, Alonsocde J. Prevalence of major depressive disorder and association with personal and socio-economic factors. Results for Spain of the European Health interview survey 2014-2015. J Affect Disord. 2018;239:203-220. doi:10.1016/j.jad.2018.06.051
Clinical Epidemiology

\section{Publish your work in this journal}

Clinical Epidemiology is an international, peer-reviewed, open access, online journal focusing on disease and drug epidemiology, identification of risk factors and screening procedures to develop optimal preventative initiatives and programs. Specific topics include: diagnosis, prognosis, treatment, screening, prevention, risk factor modification, systematic reviews, risk \& safety of medical interventions, epidemiology \& biostatistical methods, and evaluation of guidelines, translational medicine, health policies \& economic evaluations. The manuscript management system is completely online and includes a very quick and fair peer-review system, which is all easy to use.

\section{Dovepress}

